\title{
Design and Implementation of an Efficient Stack Machine
}

\author{
Martin Schoeberl \\ JOP.design \\ Vienna, Austria \\ martin@jopdesign.com
}

\begin{abstract}
Although virtually every processor today uses a loadstore register architecture, stack architectures attract attention again due to the success of Java. The intermediate language of Java, the Java bytecodes, is stack based and therefore a hardware realization of the Java Virtual Machine $(J V M)$, a Java processor, is also stack based. In this paper two different architectures, found in Java processors, are presented. Detailed analysis of the JVM access patterns to the stack prove that a simpler and faster solution is possible. The proposed solution is a stack with two levels of on-chip cache.
\end{abstract}

\section{Introduction}

The concept of a stack has a long tradition, but stack machines no longer form part of mainstream computers. Although stacks are no longer used for expression evaluation, they are still used for the context save on a function call. A niche language, Forth [5], is stack-based and known as an efficient language for controller applications. Some hardware implementations of the Forth abstract machine do exist. These Forth processors are stack machines.

The Java programming language defines not only the language but also a binary representation of the program and an abstract machine, the JVM, to execute this binary. The JVM is similar to the Forth abstract machine in that it is also a stack machine. However, the usage of the stack differs from Forth in such a way that a Forth processor is not an ideal hardware platform to execute Java programs.

In this paper, the stack usage in the JVM is analyzed. We will see that, besides the access to the top elements of the stack, an additional access path to a random element of the stack is necessary for an efficient implementation of the JVM. Two architectures will be presented for this mixed access mode of the stack. Both architectures are used in Java processors. However, we will also show that the JVM does not need a full three-port access to the stack as implemented in the two architectures. This allows for a simple and more elegant design of the stack for a Java processor. This proposed architecture will then be compared with the other two at the end of this paper.

\section{Java Computing Model}

The Java Virtual Machine (JVM) is not a pure stack machine in the sense of, for instance, the stack model in Forth. The JVM operates on a LIFO stack as its operand stack. The JVM supplies instructions to load values on the operand stack, and other instructions take their operands from the stack, operate on them and push the result back onto the stack. For example, the iadd instruction pops two values from the stack and pushes the result back onto the stack. These instructions are the stack machine's typical zeroaddress instructions. The maximum depth of this operand stack is known at compile time. In typical Java programs, the maximum depth is very small. To illustrate the operation notation of the JVM, Table 1 shows the evaluation of an expression for a stack machine notation and the JVM bytecodes.

\begin{tabular}{ll}
\multicolumn{2}{c}{$A=B+C * D$} \\
\hline Stack & JVM \\
\hline push B & iload_1 \\
push C & iload_2 \\
push D & iload_3 \\
$*$ & imul \\
+ & iadd \\
pop A & istore_0
\end{tabular}

Table 1. Standard stack notation and the corresponding JVM instructions

Instruction iload_n loads an integer value from a local variable at position $n$ and pushes the value on TOS. The 
JVM contains another memory area for method local data. This area is known as local variables. Primitive type values, such as integer and float, and references to objects are stored in these local variables. Arrays and objects cannot be allocated in a local variable, as in $\mathrm{C}++$. They have to be placed on the heap. Different instructions transfer data between the operand stack and the local variables. Access to the first four elements is optimized with dedicated single byte instructions, while up to 256 local variables are accessed with a two-byte instruction and, with the wide modifier, the area can contain up to 65536 values.

These local variables are very similar to registers and it appears that some of these locals can be mapped to the registers of a general purpose CPU or implemented as registers in a Java processor. On method invocation, local variables could be saved in a frame on a stack, different from the operand stack, together with the return address, in much the same way as in $\mathrm{C}$ on a typical processor. This would result in the following memory hierarchy:

- On-chip hardware stack for ALU operations

- A small register file for frequently-accessed variables

- A method stack in main memory containing the return address and additional local variables

However, the semantics of method invocation suggest a different model. The arguments of a method are pushed on the operand stack. In the invoked method, these arguments are not on the operand stack but are instead accessed as the first variables in the local variable area. The real method local variables are placed at higher indices. Figure 1 gives an example of the argument passing mechanism in the JVM. These arguments could be copied to the local variable area of the invoked method. To avoid this memory transfer, the entire variable area (the arguments and the variables of the method) is allocated on the operand stack. However, in the invoked method, the arguments are buried deep in the stack.

This asymmetry in the argument handling prohibits passing down parameters through multiple levels of subroutine calls, as in Forth. Therefore, an extra stack for return addresses is of no use for the JVM. This single stack now contains the following items in a frame per method:

- The local variable area

- Saved context of the caller

- The operand stack

A possible implementation of this layout is shown in Figure 2. A method with two arguments, $\arg _{-} 1$ and arg_2 (arg_0 is the this pointer), is invoked in this example. The invoked method sees the arguments as var_1 and var_2. var_3 is the only local variable of the method. SP is a pointer to the top of stack and VP points to the start of the variable area.

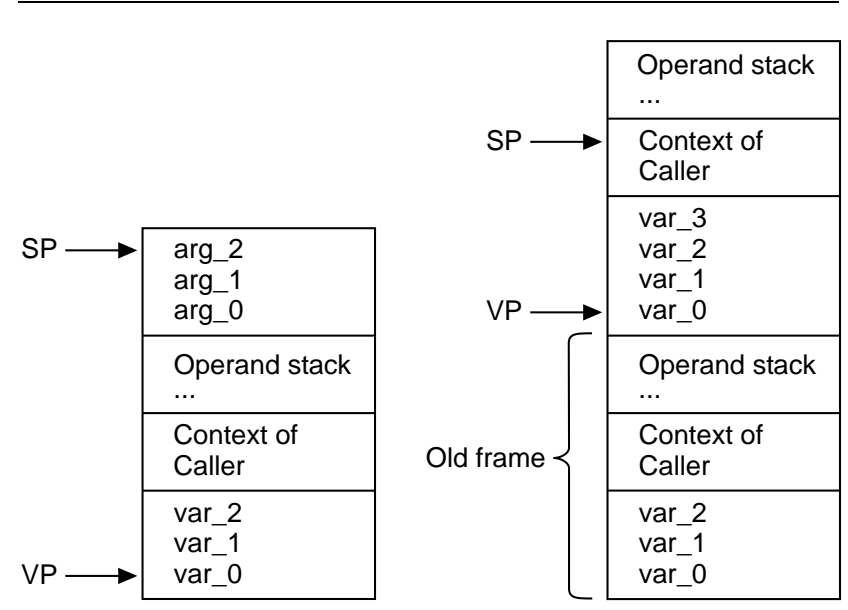

Figure 2. Stack change on method invocation

\section{Access Pattern on the Java Stack}

The pipelined architecture of a Java processor executes basic instructions in a single cycle. A stack that contains the operand stack and the local variables results in following access patterns:

Stack Operation: Read of the two top elements, operate on them and push back the result on the top of the stack. The pipeline stages for this operation are:

value $1 \leftarrow$ stack $[\mathrm{sp}]$, value $2 \leftarrow$ stack $[\mathrm{sp}-1]$

result $\leftarrow$ value1 op value2, $\mathrm{sp} \leftarrow$ sp-1

stack $[\mathrm{sp}] \leftarrow$ result

Variable Load: Read of a data element deeper down in the stack, relative to a variable base address pointer (VP), and push this data on the top of the stack. This operation needs two pipeline stages:

value $\leftarrow$ stack [vp+offset], sp $\leftarrow$ sp+1

stack $[\mathrm{sp}] \leftarrow$ value

Variable Store: Pop the top element of the stack and write it in the variable relative to the variable base address:

value $\leftarrow$ stack $[\mathrm{sp}]$

stack[vp+offset] $\leftarrow$ value, $\mathrm{sp} \leftarrow \mathrm{sp}-1$

For single cycle execution of these operations, a three-port memory or register file (two read ports and one write port) is necessary.

\section{Different Realizations of a Stack Cache}

As the stack is a heavily accessed memory region, the stack - or part of it - has to be placed in the upper level of the memory hierarchy. This part of the stack is referred to as stack cache in this paper. As described in [3], a typi- 


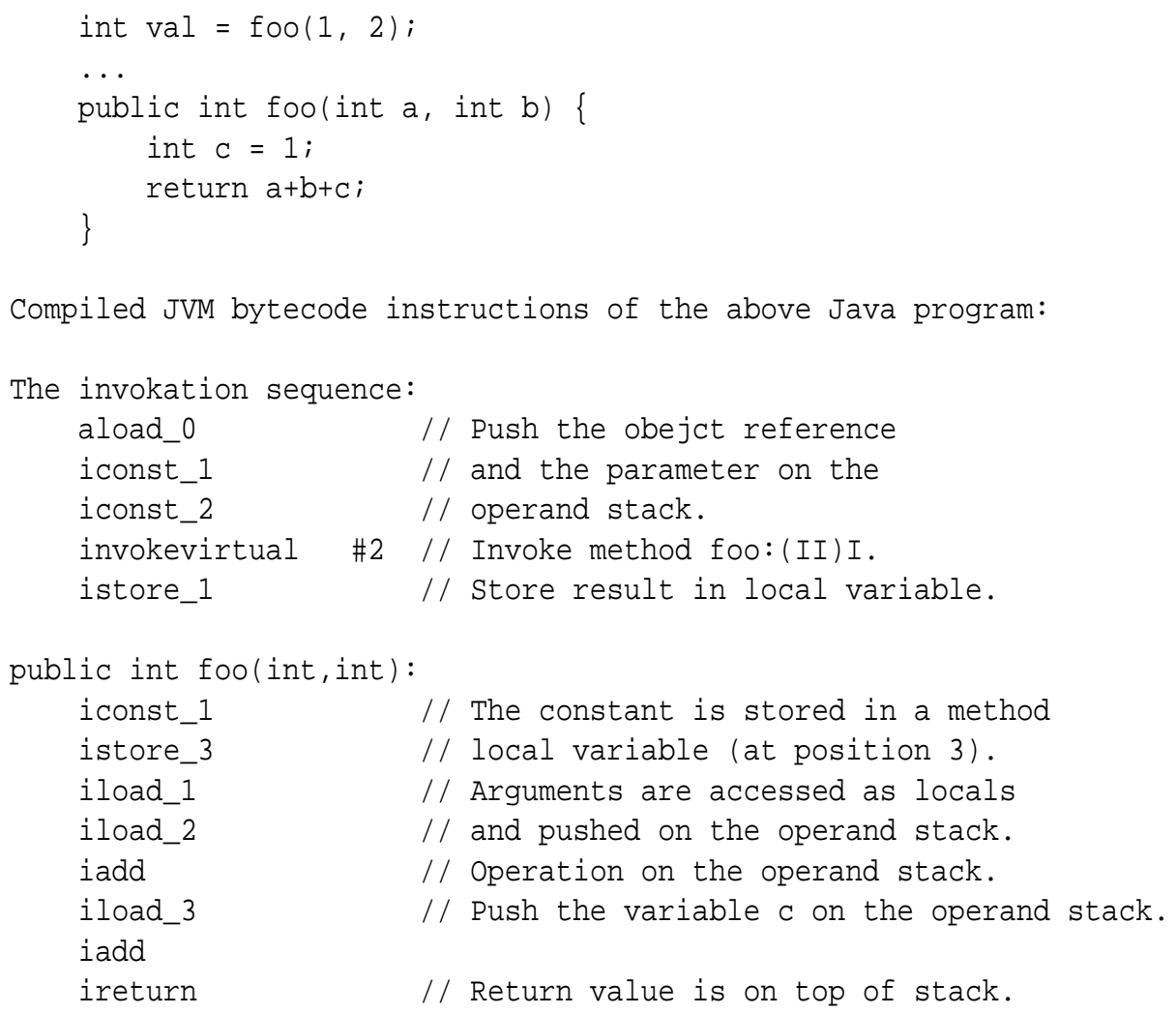

Figure 1. Example of parameter passing and access in the JVM

cal memory hierarchy contains the following elements, with increasing access time and size:

- CPU register

- On-chip cache memory

- Off-chip cache memory

- Main memory

- Magnetic disk for virtual memory

For a stack cache, a register file is the solution with the shortest access time. However, in order to store more than a few elements in the cache, an on-chip memory realization can provide a larger cache. Both variants have been used and are described below.

\subsection{The Register File as a Stack Cache}

An example of a Java processor that uses a register file is Sun's picoJava [9]. It contains 64 registers, organized as a circular buffer. To compensate for this small stack cache, an automatic spill and fill circuit needs another read/write port to the register file. aJile's JEMCore [2] is a direct-execution Java processor core that contains 24 registers. Only six of them are used to cache the top elements of the stack. With

\begin{tabular}{lc} 
Basic function & Gate count \\
\hline D-Flip-Flop & 5 \\
2:1 MUX & 3 \\
4:1 MUX & 5 \\
8:1 MUX & 9 \\
SRAM Bit & 1.5
\end{tabular}

Table 2. Simplified gate count for basic functions

this small register count, local variables are not part of the cache. The Ignite [6] (formerly known as PSC1000) is a stack processor, originally designed as a Forth processor and now promoted as a Java processor, has an operand stack that contains 18 registers with automatic spill and fill.

A basic pipeline for a stack processor with a register file contains the following stages:

1. IF - instruction fetch

2. ID - instruction decode

3. EX - read register file and execute

4. WB - write result back to register file

With this pipeline structure, a single data-forwarding path between WB and EX is necessary. The ALU with the 


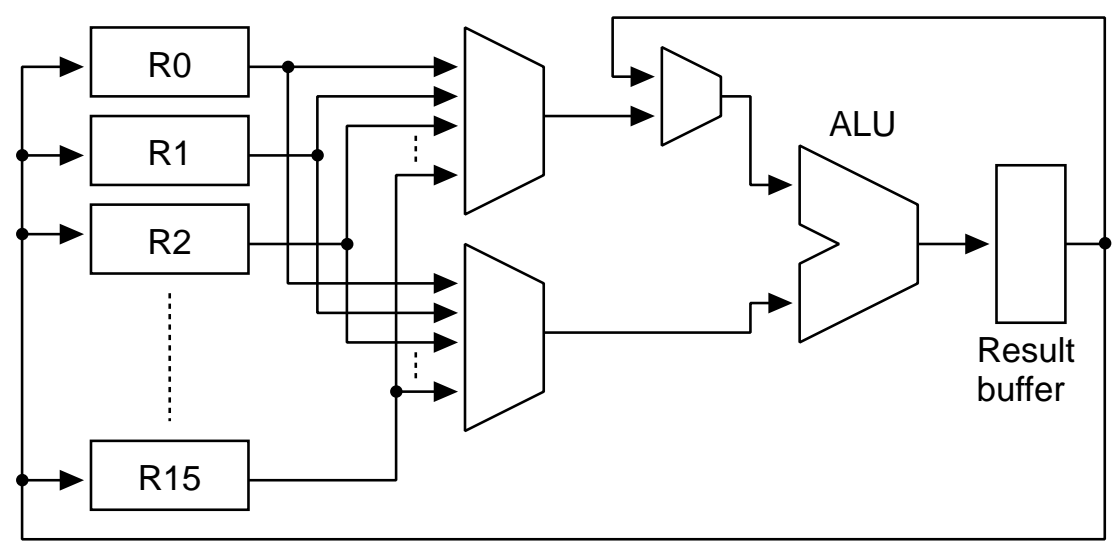

Figure 3. A stack cache with registers

register file (with a size of 16, a common size for RISC processors) and the bypass unit are shown in Figure 3. In Table 3 the hardware resources of this type of stack cache is approximated, using the values given in Table 2 (a MUX not found in this table is assumed to use combinations of the basic types; e.g. two 8:1 and one 2:1 for a 16:1). An experimental evaluation of this architecture in an FPGA is described in Section 6 .

\begin{tabular}{llr} 
Function block & Basic function & Gate count \\
\hline Register File & 512 D-Flip-Flops & 2,560 \\
Read MUX & 2x32 16:1 MUX & 1,344 \\
Forward MUX & 32 2:1 MUX & 96 \\
ALU buffer & 32 D-Flip-Flops & 160 \\
\hline Total & & 4,160
\end{tabular}

Table 3. Estimated gate count for a stack cache with registers

\subsection{On-chip Memory as Stack Cache}

Using SRAM on the chip provides a large stack cache (e.g. 128 entries). However, as we have seen in Section 3 , a three-port memory and one additional pipeline stage are necessary:

1. IF - instruction fetch

2. ID - instruction decode

3. RD - memory read

4. EX-execute

5. WB - write result back to memory
With this pipeline structure, two data forwarding paths are necessary. The resulting architecture is shown in Figure 4 and a gate count estimate is provided in Table 4. This version needs $70 \%$ more resources than the first one, but provides an eight times larger stack cache.

\begin{tabular}{llc}
\hline Function block & Basic function & Gate count \\
\hline Stack RAM & e.g. 128x32 Bits & 6,144 \\
Port buffer & 2x32 D-Flip-Flops & 320 \\
Forward MUX & 32 2:1 and 3:1 MUX & 288 \\
ALU buffer & 2x32 D-Flip-Flops & 320 \\
\hline Total & & 7,072 \\
& \\
Table 4. Estimated gate count for a stack cache \\
with on-chip RAM
\end{tabular}

Example designs that use this kind of stack cache are (i) Komodo [10], a Java processor intended as a basis for research on multithreaded real-time scheduling, and (ii) FemtoJava [4], a research project to build an application specific Java processor. Both designs avoid memory doubling by serializing the two reads. This serialization results in a minimum of a two clock cycles execution time for basic instructions or halves the clock frequency of the whole pipeline.

\section{A Two-Level Stack Cache}

In this section, we will discuss access patterns of the JVM and their implication on the functional units of the pipeline. A faster and smaller architecture is proposed for the stack cache of a Java processor. 


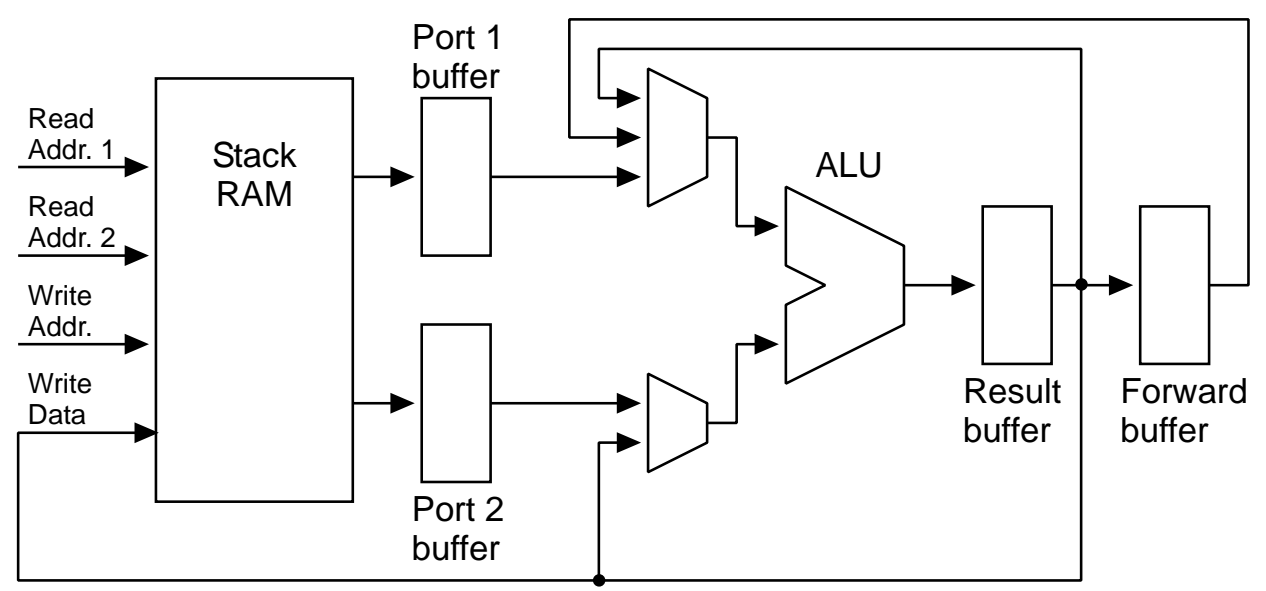

Figure 4. A stack cache with on-chip RAM

\subsection{JVM Stack Access Revised}

If we analyze the JVM's access patterns to the stack in more detail, we can see that a two-port read is only performed with the two top elements of the stack. All other operations with elements deeper in the stack, local variables load and store, only need one read port. If we only implement the two top elements of the stack in registers, we can use a standard on-chip RAM with one read and one write port.

We will show that all operations can be performed with this configuration. Let $A$ be the top-of-stack, $B$ the element below top-of-stack. The memory that serves as the second level cache is represented by the array $s m$. Two indices in this array are used: $p$ points to the logical third element of the stack and changes as the stack grows or shrinks, $v$ points to the base of the local variables area in the stack and $n$ is the address offset of a variable. $o p$ is a two operand stack operation with a single result (i.e. a typical ALU operation).

Case 1: ALU operation

$$
\begin{aligned}
& A \leftarrow A \text { op } B \\
& B \leftarrow s m[p] \\
& p \leftarrow p-1
\end{aligned}
$$

The two operands are provided by the two top level registers. A single read access from $s m$ is necessary to fill $B$ with a new value.

Case 2: Variable load (Push)

$$
\begin{aligned}
& A \leftarrow s m[v+n] \\
& B \leftarrow A \\
& s m[p+1] \leftarrow B \\
& p \leftarrow p+1
\end{aligned}
$$

One read access from $s m$ is necessary for the variable read. The former TOS value moves down to $B$ and the data previously in $B$ is written to $s m$.
Case 3: Variable store (Pop)

$$
\begin{aligned}
& s m[v+n] \leftarrow A \\
& A \leftarrow B \\
& B \leftarrow s m[p] \\
& p \leftarrow p-1
\end{aligned}
$$

The TOS value is written to $s m$. $A$ is filled with $B$ and $B$ is filled in an identical manner to Case 1, needing a single read access from $s m$.

We can see that all three basic operations can be performed with a stack memory with one read and one write port. Assuming a memory is used that can handle concurrent read and write access, there is no structural access conflict between $A, B$ and $\mathrm{sm}$. That means that all operations can be performed concurrently in a single cycle.

As we can see in Figure 2 the operand stack and the local variables area are distinct regions of the stack. A concurrent read from and write to the stack is only performed on a variable load or store. When the read is from the local variables area the write goes to the operand area; a read from the operand area is concurrent with a write to the local variables area. Therefore there is no concurrent read and write to the same location in $\mathrm{sm}$. There is no constraint on the read-during-write behavior of the memory (old data, undefined or new data), which simplifies the memory design. In a design where read and write-back are located in different pipeline stages, as in the architectures described above, either the memory must provide the new data on a readduring-write, or external forward logic is necessary.

From the three cases described, we can derive the memory addresses for the read and write port of the memory, as shown in Table 5 .

\subsection{The Datapath}

The architecture of the two-level stack cache can be seen 


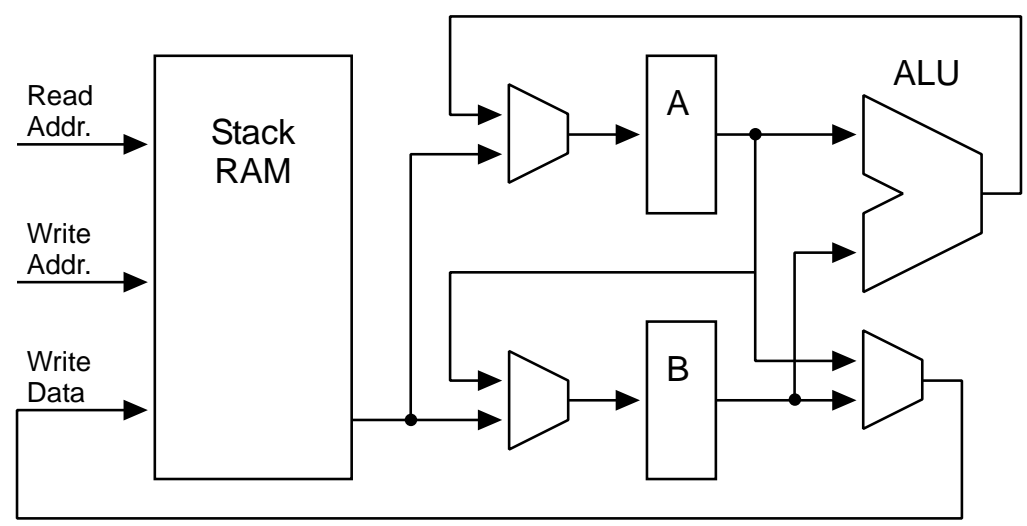

Figure 5. Two-level stack cache

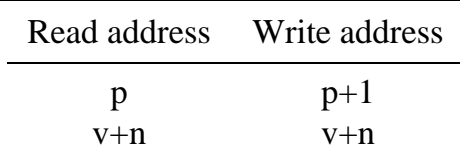

Table 5. Memory addresses

in Figure 5, Register $A$ represents the top-of-stack and register $B$ the data below the top-of-stack. ALU operations are performed with these two registers and the result is placed in $A$. During such an ALU operation, $B$ is filled with new data from the stack RAM. A new value from the local variable area is loaded directly from the stack RAM into $A$. The data previously in $A$ is moved to $B$ and the data from $B$ is spilled to the stack RAM. $A$ is stored in the stack RAM on a store instruction to the local variable. The data from $B$ is moved to $A$ and $B$ is filled with a new value from the stack RAM. All these operations are performed concurrently in one cycle.

With this architecture, the pipeline can be reduced to three stages:

1. IF - instruction fetch

2. ID - instruction decode

3. EX - execute, load or store

The estimated resource usage of this two-level stack cache architecture is given in Table 6. It can be seen that this architecture is roughly as complex as the solution given above (about 5\% less gates). However, the reduced complexity with the two-port RAM instead of a three port RAM is not included in the table. The critical path through the ALU contains only one 2:1 MUX to register $A$ in this solution, rather than one 3:1 MUX in one ALU path and one 2:1 MUX in the other ALU path. As no data forwarding logic is necessary, the decoding logic is also simpler.

\begin{tabular}{llr}
\hline Function block & Basic function & Gate count \\
\hline Stack RAM & e.g. 128x32 Bits & 6,144 \\
TOS, TOS-1 buffer & 2x32 D-Flip-Flops & 320 \\
Three MUX & 3x32 2:1 MUX & 288 \\
\hline Total & & 6,752
\end{tabular}

Table 6. Estimated gate count for the two-level stack cache

\subsection{Data Forwarding - A Non Issue}

Data dependencies in the instruction stream result in the so-called data hazards [3] in the pipeline. Data forwarding is a technique that moves data from a later pipeline stage back to an earlier one to solve this problem. The term forward is correct in the temporal domain as data is transferred to an instruction in the future. However, it is misleading in the structural domain as the forward direction is towards the last pipeline stage for an instruction.

As the probability of data dependency is very high in a stack-based architecture, one would expect several data forwarding paths to be necessary. However, in the two-level architecture proposed, with its resulting three-stage pipeline, no data hazards will occur and no data forwarding is therefore necessary. This simplifies the decoding stage and reduces the number of multiplexers in the execution path. We will show that none of the three data hazard types [3] are an issue in this architecture. With instructions $i$ and $j$, where $i$ is issued before $j$, the data hazard types are:

Read after write: $j$ reads a source before $i$ writes it. This is the most common type of hazard and, in the architectures described above, is solved by using the ALU buffers and the forwarding multiplexer in the ALU datapath. On a stack architecture, write takes three forms: 
- Implicit write of TOS during an ALU operation

- Write to the TOS during a load instruction

- Write to an arbitrary entry of the stack with a store instruction

A read also occurs in three different forms:

- Read two top values from the stack for an ALU operation

- Read TOS for a store instruction

- Read an arbitrary entry of the stack with the load instruction

With the two top elements of the stack as discrete registers, these values are read, operated on and written back in the same cycle. No read that depends on TOS or TOS-1 suffers from a data hazard. Read and write access to a local variable is also performed in the same pipeline stage. Thus, the read after write order is not affected. However, there is also an additional hidden read and write - the fill and spill of register $\mathrm{B}$ :

- $B$ fill: $B$ is written during an ALU operation and on a variable store. During an ALU operation, the operands are the values from $A$ and the old value from $B$. The new value for $B$ is read from the stack memory and does not depend on the new value of $A$. During a variable store operation, $A$ is written to the stack memory and does not depend on $B$. The new value for $B$ is also read from the stack memory and it is not obvious that this value does not depend on the written value. However, the variable area and the operand stack are distinct areas in the stack (this changes only on method invocation and return), guaranteeing that concurrent $\mathrm{read} /$ write access does not produce a data hazard.

- $B$ spill: $B$ is read on a load operation. The new value of $B$ is the old value of $A$ and does not therefore depend on the stack memory read. $B$ is written to the stack. For the read value from the stack memory that goes to $A$, the argument concerning the distinct stack areas in the case of $B$ fill described above still applies.

Write after read: $j$ writes a destination before it is read by $i$. This cannot take place as all reads and writes are performed in the same pipeline stage keeping the instruction order.

Write after write: $j$ writes an operand before it is written by $i$. This hazard is not present in this architecture as all writes are performed in the same pipeline stage.

\section{Resource Usage Compared}

The three architectures described above are implemented in an FPGA (Altera's EP1C6Q240C6 [1]). The three-port memory for the second solution is emulated with two embedded memory blocks. The ALU for this comparison is kept simple with the following functions: NOP, ADD, SUB, POP, AND, OR, XOR and load external data. The load of external data is necessary in order to prevent the synthesizer from optimizing away the whole design. A real implementation of an ALU for a Java processor [7] is a little bit more complex with a barrel shifter and additional load pathes. In order to gain the maximum operating frequency for the design, the testbed for this architecture contains registers for the external data, the RAM address busses, and the control and select signals. Table 7 shows the resource usage and maximum operation frequency of the three different architectures.

LC stands for 'Logic Cell' and is the basic element in an FPGA: a 4-bit lookup table with a register. The LC count in the table includes the register count. The ALU alone without any stack cache needs 194 LCs. In the first line, the testbed is combined with the ALU without any stack caching, as a reference design. With this configuration, we can obtain the maximum possible speed of the registered ALU in this FPGA technology, in this case an operating frequency of $237 \mathrm{MHz}$ or a $4.2 \mathrm{~ns}$ delay. This value is an upper bound of the system frequency. Every pipelined architecture needs one or more multiplexer in the ALU path, either for data forwarding or for operand selection, resulting in a longer delay. The fourth and fifth columns represent the resource usage of the cache logic without the testbed and ALU. The last column shows the effective cache size in data words.

The version with the 16 registers was synthesized with two different synthesizer settings. In the first setting, the register file is implemented with discrete registers while, with a different setting, the register file is automatically implemented in two 32-bits embedded RAM blocks. Two different RAM blocks are necessary to provide two read ports and one write port. In both versions, the delay time to read the register file (delay through the 16:1 MUX of $4.9 \mathrm{~ns}$ or RAM access time of $4.6 \mathrm{~ns}$ ) is in the same order as the delay time through the ALU, resulting in a system frequency of half the theoretical frequency of that with the ALU alone. As the structure of the version with the embedded RAM block is very similar with the SRAM cache, only the version with the discrete registers is shown in Table 7.

The stack cache with a RAM and registers on the RAM output (the additional pipeline stage) performs better than the first solution. However, the 3:1 MUX in the critical path still adds $2.3 \mathrm{~ns}$ to the delay time. Compared with the proposed solution (in the last line), we see that double the amount of RAM is needed for the two read ports.

The two-level stack cache solution performs at $213 \mathrm{MHz}$, i.e. almost the theoretical system frequency (in practice, about $10 \%$ slower). Only a 2:1 MUX is added to the crit- 


\begin{tabular}{|c|c|c|c|c|c|c|c|}
\hline \multirow[t]{2}{*}{ Design } & \multicolumn{2}{|c|}{ Total } & \multicolumn{2}{|c|}{ Cache } & \multirow{2}{*}{$\begin{array}{c}\text { Memory } \\
\text { [bit] }\end{array}$} & \multirow{2}{*}{$\begin{array}{c}\text { fmax } \\
{[\mathrm{MHz}]}\end{array}$} & \multirow{2}{*}{$\begin{array}{c}\text { Size } \\
\text { [word] }\end{array}$} \\
\hline & $\mathrm{LCs}$ & Registers & $\mathrm{LCs}$ & Registers & & & \\
\hline Testbed with ALU & 261 & 166 & - & - & - & 237 & - \\
\hline 16 register cache & 968 & 657 & 707 & 491 & 0 & 110 & 16 \\
\hline SRAM cache & 372 & 185 & 111 & 19 & 8,192 & 153 & 128 \\
\hline Two-level cache & 373 & 184 & 112 & 18 & 4,096 & 213 & 130 \\
\hline
\end{tabular}

ical path. The single read port memory only needs half the number of memory bits of the other two solutions.

\section{Conclusion}

In this paper, the stack architecture of the JVM was analyzed. We have seen that the JVM is different from the classical stack architecture. The JVM uses the stack both as an operand stack and as the storage place for local variables. Local variables are placed in the stack at a deeper position. To load and store these variables, a random access path to the stack is necessary. As the stack is the most frequent accessed memory area in the JVM, caching of this memory is mandatory for a high-performing Java processor.

A common solution, found in a number of different Java processors, is to implement this stack cache as a standard three-port register file with additional support to address this register file in a stack like manner. The architectures presented above differ in the realization of the register file: as a discrete register or in on-chip memory. Implementing the stack cache as discrete registers is very expensive. A three-port memory is also an expensive option for an ASIC and unusual in an FPGA. It can be simulated in an FPGA by two memories with a single read and write port. The write data is written in both memory blocks and each memory block provides a different read port. However, this also doubles the amount of memory.

Detailed analysis of the access patterns to the stack showed that only the two top elements of the stack are accessed in a single cycle. Given this fact, the proposed architecture uses registers to cache only the two top elements of the stack. The next level of the stack cache is provided by a simple on-chip memory. The memory automatically spills and fills the second register. Implementing the two top elements of the stack as fixed registers, instead of elements that are indexed by a stack pointer, also greatly simplifies the overall pipeline. This architecture is successfully implemented in a Java Optimized Processor (JOP) [8].

The proposed stack architecture has the following advantages: (i) Simpler cache memory results in having half the memory usage of the other solutions in an FPGA. (ii) Minimal impact in the raw speed of the ALU. Operates at almost the theoretical maximum system frequency of the ALU. (iii)
Single read, execute and write-back pipeline stage results in an overall 3-stage pipeline processor design. (iv) No data forwarding is necessary, which simplifies instruction decode logic and reduces the multiplexer count in the critical path.

\section{References}

[1] A. Corporation. Cyclone FPGA Family Data Sheet, ver. 1.2, April 2003.

[2] D. Hardin. Real-Time Objects on the Bare Metal: An Efficient Hardware Realization of the JavaTM Virtual Machine. In Proceedings of the Fourth International Symposium on Object-Oriented Real-Time Distributed Computing, page 53. IEEE Computer Society, 2001.

[3] J. Hennessy and D. Patterson. Computer Architecture: A Quantitative Approach, 3rd ed. Morgan Kaufmann Publishers Inc., Palo Alto, CA 94303, 2002.

[4] S. Ito, L. Carro, and R. Jacobi. Making Java work for microcontroller applications. IEEE Design \& Test of Computers, 18(5):100-110, 2001.

[5] P. Koopman. Stack Computers: The New Wave. Ellis Horwood, 1989. Out of print, now available over the internet.

[6] PTSC. Ignite processor brochure, rev 1.0. Available at http://www.ptsc.com.

[7] M. Schoeberl. JOP: A Java optimized processor. In R. Meersman, Z. Tari, and D. Schmidt, editors, On the Move to Meaningful Internet Systems 2003: Workshop on Java Technologies for Real-Time and Embedded Systems (JTRES 2003), volume 2889 of Lecture Notes in Computer Science, pages 346-359, Catania, Italy, November 2003. Springer.

[8] M. Schoeberl. Java technology in an FPGA. In Proceedings of the International Conference on Field-Programmable Logic and its applications (FPL 2004), Antwerp, Belgium, August 2004.

[9] Sun. picoJava-II Microarchitecture Guide. Sun Microsystems, March 1999.

[10] R. Zulauf. Entwurf eines Java-Mikrocontrollers und prototypische Implementierung auf einem FPGA. Master's thesis, University of Karlsruhe, 2000. 